\title{
A fast approach coupling Boundary Element Method and plane wave approximation for wave interaction analysis in sparse arrays of wave energy converters
}

\author{
Jitendra Singh *, Aurélien Babarit \\ LUNAM Université, Ecole Centrale de Nantes-CNRS, 1 rue de la Noe, 44300 Nantes Cedex 3, France
}

\begin{abstract}
A computational approach is developed to investigate wave interaction effects in sparse arrays of floating bodies (such as wave energy converters) based on linear potential theory. In particular, the wave diffraction and radiation problems in a multiple body array are solved in reasonable time and accuracy. In contrast to previous approaches that have considered all bodies in the array as a single module, the present approach treats each body in the array as an isolated body. The interactions resulting from the scattered wave field among the bodies are then taken into account via plane wave approximation in an iterative manner. The boundary value problem corresponding to an isolated body is solved by the Boundary Element Method (BEM). The approach is useful for wave periods in the range 4-15 s, provided that the bodies are separated by at least five times the characteristic dimension of a body. The main advantage of the approach is that the computational time and memory requirements are significantly less than that of conventional BEM. In this paper, first, the numerical results for hydrodynamic coefficients computed by the proposed approach are validated against conventional BEM. Next, the wave interaction effects on power production are investigated in arrays of 50 wave energy converters.
\end{abstract}

\section{Introduction}

This study investigates wave interaction effects in arrays of oscillating wave energy converters (WECs). It is anticipated that for power production at a commercial scale, tens of WECs would be deployed in arrays, the so-called WEC farms. Therefore, in order to make reliable estimates of energy production, it becomes necessary to take into account wave interaction effects. This aspect has been covered in many studies, c.f. Budal (1977), Falcaode (2002), Cruz et al. (2009), Ricci et al. (2007), Babarit (2010) and Borgarino et al. (2012), in regular and irregular wave fields for a variety of WECs. In developing oscillating WEC arrays with device dimensions $\sim 10-20 \mathrm{~m}$, the distance between the devices could be as much as a few hundred meters due to practical considerations, such as mooring, installation and maintenance issues. Consequently, this study investigates wave interaction effects in such sparse WEC arrays, where the ratio of separating distance to device dimension is large.

A viable approach to solving full hydrodynamic interaction phenomena in a multiple body array is to model the problem

\footnotetext{
* Corresponding author. Current address: IBM Research Collaboratory, Singapore. E-mail addresses: jsingh@sg.ibm.com (J. Singh), aurelien.babarit@ec-nantes.fr (A. Babarit).
}

using linear potential theory. This requires solving the diffraction and radiation problems over the frequency domain; the hydrodynamic coefficients (excitation force, added mass and wave damping) can then be easily post-processed. A judicious choice for solving these problems is the Boundary Element Method (BEM), since the domain is unbounded and only discretization of the boundary is required. However, when the array consists of several bodies, the number of discretization elements increases, and use of the BEM becomes prohibitive due to the computational requirements of solving a dense linear matrix system. In addition, a comprehensive analysis requires that the solution of diffraction and radiation problems be sought at several frequencies in the range of interest, thereby further increasing the computational time requirements. It is worth noting that developing fast algorithms to solve dense matrix system in BEM is an active area of research. There have been successful attempts to accelerate the BEM by coupling it with fast methods, such as fast multipole methods (FMM) (Greengard and Rokhlin, 1987), pre-corrected fast Fourier transforms (FFT) (Phillips and White, 1997) and other methods. In the field of wave hydrodynamics, the BEM-FMM coupling for solving diffraction and radiation problems has been used in Utsunomiya and Watanabe (2002), Teng and Gou (2006), and Borgarino et al. (2011) for specific applications. Acceleration using fast Fourier transforms has been performed in Kring et al. (2000) and others. However, these approaches have some limitations. In FMM, 
numerical convergence of the existing multipole series expansion of the free surface Green's function can be difficult, thereby limiting the scope of developing this approach for generic three-dimensional bodies; whereas, in FFT methods, construction of a grid and projection operations over the full domain of the sparse array would lead to a sub-optimal algorithm.

Besides the aforementioned fast methods, interaction approaches have also received a significant attention, particularly for axisymmetric bodies. A multiple scattering theory (Ohkusu, 1974) combined with a direct matrix method has been developed in Kashiwagi (2000). An extension of this theory, the so-called hierarchical interaction theory, has been presented in finite (Kagemoto and Yue, 1986) and infinite depths (Peter and Meylan, 2004). The plane wave approximation (PWA), also known as wide space approximation, coupled with direct matrix approach has been proposed in Simon (1982). The PWA approach is based on the assumption that waves diffracted by or radiated from a body can be approximated at large distances by a plane wave. This approach has been further improved to reduce the spacing requirements by taking into account non-plane correction terms in the formulation (McIver and Evans, 1984). A comparison of the multiple scattering and the PWA approaches for arrays consisting of five vertical cylinders with varying radii and separating distances has been presented in Mavrakos and Mclver (1997), in which it was deduced that the PWA provides very good results for all the hydrodynamic coefficients in comparison to those provided by multiple scattering approach when the ratio of separating distance to body dimension is greater than five.

In this study, we propose a simple approach for estimating the wave interaction effects in sparse arrays of a large number of bodies of arbitrary shape. The approach couples the efficiency of the BEM with the core idea of PWA mentioned above. Essentially, when the bodies are sufficiently distant in an array, the effects of the wave field emanating from one body are taken into account by the other body as an additional plane incident wave. Here we use this approximation efficiently and develop a fast approach. The computational time and memory requirements using the proposed approach are significantly less than the conventional BEM (CBEM), and the approach is applicable to bodies of arbitrary shape. Furthermore, the approach is simple and can be easily implemented in existing diffraction/radiation solvers.

The remainder of the paper is organized as follows. We begin by recalling in Section 2 the problem formulation in potential theory and detail the necessary equations that must be solved to calculate power extraction by a WEC array. In Section 3, we illustrate our approach of coupling BEM with PWA, and in Section 4 we present numerical results to validate the approach. Section 5 analyze wave interaction effects on power production in WEC arrays. The final section presents some concluding remarks.

\section{Problem statement}

The wave interaction phenomenon in a multiple body array is modeled within the framework of linearized potential theory. Specifically, the fluid is inviscid and incompressible and the flow is irrotational. The wave amplitude and body motions are small with respect to the wavelength and body dimensions, respectively. Under these assumptions, the problem can be formulated in terms of a velocity potential, $\Phi$, satisfying the Laplace equation in the fluid domain with appropriate boundary conditions. For simplicity, we consider that the fluid domain is of infinite depth and unbounded in horizontal directions. The motion is time harmonic with circular frequency $\omega$, i.e. $\Phi(x, y, z, t)=\mathfrak{R}\left\{\phi(x, y, z) e^{-i \omega t}\right\}$. Linearization allows the velocity potential $\phi$ to be expressed as sum of the incident potential $\phi_{i n}$, the diffraction potential $\phi_{d}$ and the radiation potential $\phi_{r}$. The explicit form of the incident potential is

$\phi_{\text {in }}=\frac{g A}{\omega} e^{k z} e^{i k(x \cos \beta+y \sin \beta)}$

where $k=\omega^{2} / g$ is the wave number, $g$ is the acceleration due to gravity, $A$ is the wave amplitude and $\beta$ is the angle between the direction of propagation of the incident wave and the positive $x$ axis. The diffraction and radiation potentials correspond to the potentials generated in response to the incident waves and the fluid disturbance due to the motions of the bodies in still water, respectively. The diffraction and radiation boundary value problems can be summarized as follows:

- Diffraction problem:

$$
\begin{cases}\Delta \phi_{d}=0 & \text { in the fluid domain } \\ \nabla \phi_{d} \rightarrow 0 & z \rightarrow-\infty \\ \frac{\partial \phi_{d}}{\partial z}-k \phi_{d}=0 & \text { at mean free surface position } z=0 \\ \frac{\partial \phi_{d}}{\partial n}=-\frac{\partial \phi_{i n}}{\partial n} & \text { on mean wetted body surface }\end{cases}
$$

- Radiation problems:

$$
\begin{cases}\Delta \phi_{r_{i}}^{j}=0 & \text { in the fluid domain } \\ \nabla \phi_{r_{i}}^{j} \rightarrow 0 & z \rightarrow-\infty \\ \frac{\partial \phi_{r_{i}}^{j}}{\partial z}-k \phi_{r_{i}}^{j}=0 & \text { at mean free surface position } z=0 \\ \frac{\partial \phi_{r_{i}}^{j}}{\partial n}=n_{i}^{j} & \text { on mean wetted body surface }\end{cases}
$$

where indices $i$ and $j$ correspond to motion in any of the six degrees of freedom and the numeration of the body $(j=1, \ldots, N)$, respectively. Using this convention, $n_{i}^{j}$ denotes the component of the normal vector in the direction of motion on body $j$.

Having solved the set of diffraction and radiation problems, it is straightforward to compute the hydrodynamic coefficients: excitation force $F_{e x}(\omega)$, added mass $A M(\omega)$ and wave damping $B(\omega)$. Further, to compute the motion of a system of $N$ floating bodies for unit wave amplitude and wave frequency $\omega$ we solve

$(M+A M(\omega)) \ddot{X}+\left(B_{\text {PTO }}+B(\omega)\right) \dot{X}+\left(K_{H}+K_{\text {PTO }}\right) X=F_{\text {eX }}(\omega)$

where $X$ is the position vector, $X=\mathfrak{R}\left(\bar{X} e^{-i \omega t}\right), \dot{X}$ and $\ddot{X}$ being, respectively, the velocity and acceleration vectors. $M$ and $K_{H}$ are the mass and hydrostatic matrices of the system. An idealized power take off (PTO) is considered in this study, composed of a linear spring and damper system with stiffness $k_{\text {Рто }}$ and damping coefficient $b_{\text {Рто. These are the diagonal elements of the stiffness }}$ and damping matrices in (4), i.e. $K_{P T O_{i i}}=k_{P T O}$ and $B_{P T O_{i i}}=b_{P T O}$.

In regular waves, the mean power extracted by each device in the array per unit square of wave amplitude is

$p_{i}(\omega)=\frac{1}{2} B_{\text {РТО }} \omega^{2}\left|X_{i}\right|^{2}$.

For the whole array, the mean power is simply the sum of the mean power from each of the individual devices. For irregular waves, characterized by a wave energy spectrum $S$ (we use a standard Jonswap spectrum with frequency spreading parameter $\gamma=3.3$ ), the mean power extracted is

$P_{i}\left(H_{s}, T_{p}\right)=\int_{0}^{\infty} S\left(H_{s}, T_{p}, \omega\right) p_{i}(\omega) d \omega$

where $H_{s}$ is the significant wave height and $T_{p}$ is the peak period. The yearly average power of a body $i$, given the probability of 
occurrence $C\left(H_{s}, T_{p}\right)$ of sea state $H_{s}, T_{p}$, is calculated by

$\left\langle P_{i}\right\rangle=\sum_{H_{s}, T_{p}} P_{i}\left(H_{s}, T_{p}\right) C\left(H_{s}, T_{p}\right)$

For the whole array, the mean absorbed power is again simply the sum over all the bodies.

\section{Solution procedure}

The most time-consuming task is to solve the diffraction and radiation problems (2) and (3), usually by the BEM. It is straightforward to transform (2) and (3) into boundary integral equations, either by using Green's identity involving potentials only (direct formulation), or involving particular source distribution (indirect formulation). We prefer the source formulation, because both the diffraction and radiation problems can be formulated in a common framework as a Fredholm equation of the second kind and implemented in the software AQUAPLUS (Delhommeau, 1993). First, the following integral equation is solved for the unknown source distribution $\sigma$ on the body surfaces $S=\bigcup_{k=1}^{N} S_{k}$ :

$\frac{\sigma(\mathbf{x})}{2}-\frac{1}{4 \pi} \int_{S} \sigma\left(\mathbf{x}^{\prime}\right) \frac{\partial G\left(\mathbf{x}^{\prime}, \mathbf{x}\right)}{\partial n_{\mathbf{x}}} d S\left(\mathbf{x}^{\prime}\right)=\left\{\begin{array}{l}-\frac{\partial \phi_{i n}}{\partial n}(\mathbf{x}) \\ n_{i}^{j}(\mathbf{x})\end{array}\right.$,

where $\mathbf{x}^{\prime}$ and $\mathbf{x}$ are the source and the field point, respectively. The function $G\left(\mathbf{x}^{\prime}, \mathbf{x}\right)$ is the free surface Green's function taking into account the linearized free surface conditions, conditions at the sea bottom and radiation conditions at infinity (Newman, 1985). Having solved (8) for the density $\sigma$, the velocity potential can be computed at any arbitrary point by the following equation:

$\phi(\mathbf{x})=-\frac{1}{4 \pi} \int_{S} \sigma\left(\mathbf{x}^{\prime}\right) G\left(\mathbf{x}^{\prime}, \mathbf{x}\right) d S\left(\mathbf{x}^{\prime}\right)$.

Let us point out that the discretization of (8) leads us to a dense matrix system. For an array consisting of $N$ bodies, the number of diffraction and radiation problems to be solved for motion in one degree of freedom is $(1+N) n_{f}$, where $n_{f}$ is the number of frequencies. When $N$ is large, it follows that the number of boundary elements representing the body surface is large, and so it is not feasible to solve the problems using CBEM in reasonable time. Keeping this in mind, we now describe a simple and fast approach to solve the radiation and diffraction problems by coupling BEM with PWA.

\subsection{BEM and plane wave approximation coupling strategy}

We now describe how to take into account interactions using PWA when the bodies are sufficiently well separated. For simplicity, we first consider the interaction phenomenon in an array of two bodies. Due to incident waves (diffraction problem) or independent motion (radiation problem), body 1 scatters waves that excite body 2 . Body 2 responds to this excitation and in turn sends outgoing waves towards body 1 , which also responds to this excitation, and so on. In the same vein, body 2 interacts with body 1 due to incident waves or independent motion of the former. In what follows, as demonstrated by Simon (1982), we can approximate the impact of outgoing waves from a body on all other bodies by an incident plane wave of appropriately chosen amplitude. From a visual perspective, it can be observed that far from the generating body the outgoing waves have small curvature and they act as plane waves on the incident body. However, for numerical accuracy the criterion 'sufficiently far' requires careful stipulation. On the basis of our numerical experiments, we can state that when the separating distance between the bodies is greater than approximately five times the characteristic dimension of the body, the approximation provides reliable results for wave period ranging 4-15 s. The algorithm based on the above can now be formulated for an $\mathrm{N}$-body array in three steps, which are described below:

- Step 1: Contributions of individual bodies. In this step, we compute the potential on each body by considering them to be isolated, i.e. hypothetically assuming that the bodies have no interaction with each other. The computation required is straightforward: solve integral Eqs. (8) and (9) on the wetted body surface of each individual body. In the first iteration, the usual boundary conditions in (2) and (3) for diffraction and radiation problems are assigned locally to the body. The boundary conditions for the subsequent iterations, taking into account wave interactions, are obtained in step 2. It is worth noting that there is an additional advantage when bodies are of the same shape: the influence matrices corresponding to Green's function, and the inverse of the left-hand side of (8) corresponding to a single body, are computed once and stored, to be used for all other bodies when their contribution is sought.

- Step 2: Contributions due to wave interaction. As already stated, we approximate the potential due to body $i$ on body $j$ by the potential of an incident plane wave. Since we know the source distribution on an arbitrary body $i$ from step 1 , the potential at the mean center position of all other bodies $\left(x_{j}, y_{j}, 0\right)(j \neq i)$ due to sources on body $i$ is computed by solving (9); this is denoted by $\phi_{i j}$. The total effect at body $j$ is taken as the sum of the effects from all other bodies in terms of incident plane waves of complex amplitude $\phi_{i j}$, i.e.

$\phi_{j}^{*}=\sum_{i=1}^{N} \phi_{i j} e^{k z} e^{\mathrm{i} k\left(\left(x-x_{j}\right) \cos \theta_{i j}+\left(y-y_{j}\right) \sin \theta_{i j}\right)}, \quad j \neq i$,

where $\theta_{i j}$ is the angle subtended by the mean center position of body $i$ while considering the origin to be the mean center position of body $j$ (Fig. 1). Having computed this effect at each body in the array, we then compute the contribution of all the bodies as isolated (as in step 1) due to the excitation induced by $\phi^{*}$.

- Step 3: Check for convergence. At each iteration, steps 1 and 2 contribute to the total potential on the body surface. This process is repeated until either the amplitude of the incident waves $\left|\phi_{i j}\right|$ becomes smaller than $10^{-2}$ for all bodies, or the maximum number of iterations is reached. This maximum is set to twice the number of bodies in the array. A shortcoming of the approach is that there may be resonance effects for some frequencies, resulting in non-convergence of the iterative procedure. This situation is handled by making a simple

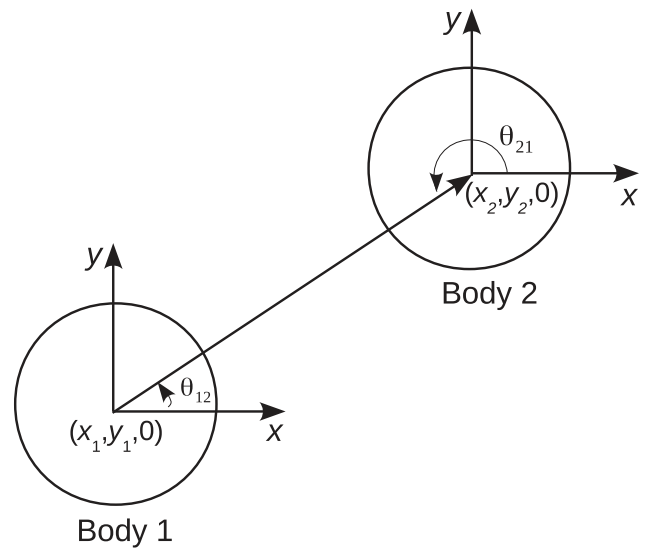

Fig. 1. Notation of the interacting bodies. 
treatment that provide reasonably accurate results, namely that at any given iteration, if the amplitude $\left|\phi_{i j}\right|$ is larger than the previous two iterations, we stop including the interactions from body $j$ on body $i$.

\section{Test arrays}

Here, we present numerical results to validate the approach. For this purpose, two arrays of 25 heaving cylinders and 25
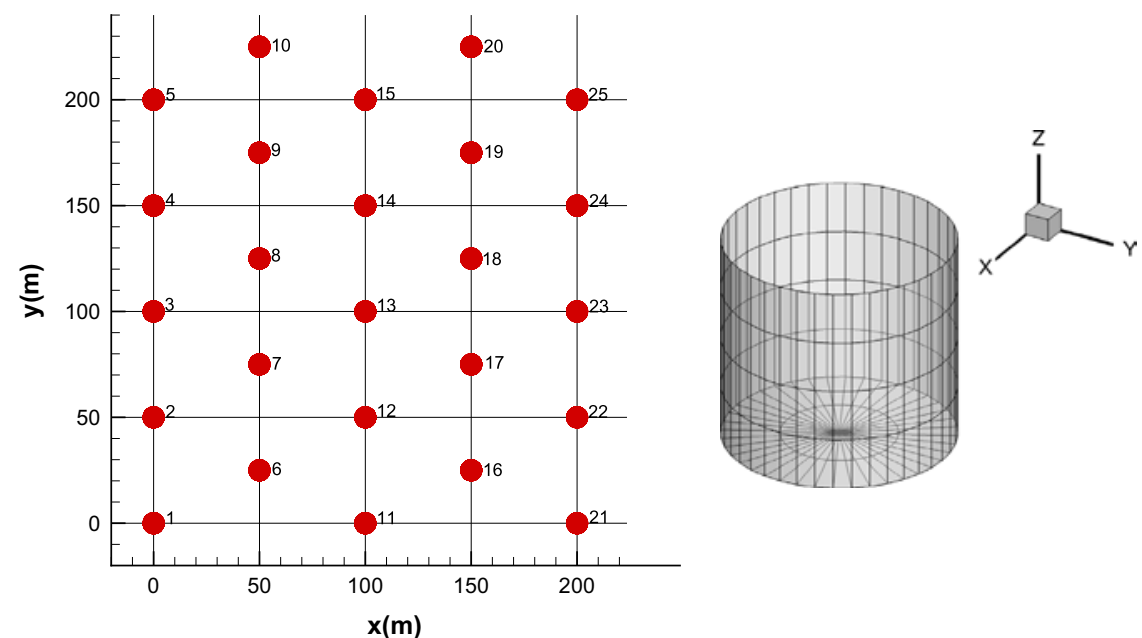

Fig. 2. Top view of array layout and surface mesh of a cylinder.
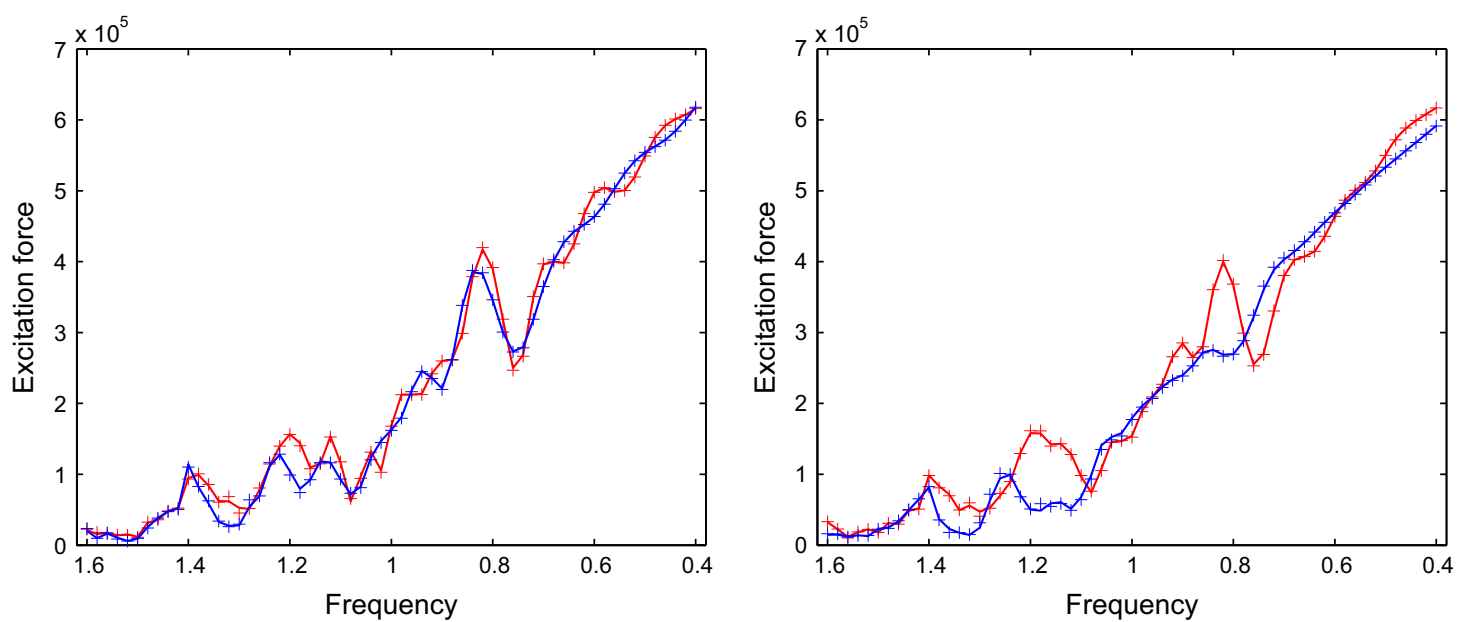

Fig. 3. Left: excitation forces on cylinders 2 (red) and 9 (blue). Right: excitation forces on cylinders 5 (red) and 24 (blue). (For interpretation of the references to color in this figure caption, the reader is referred to the web version of this paper.)
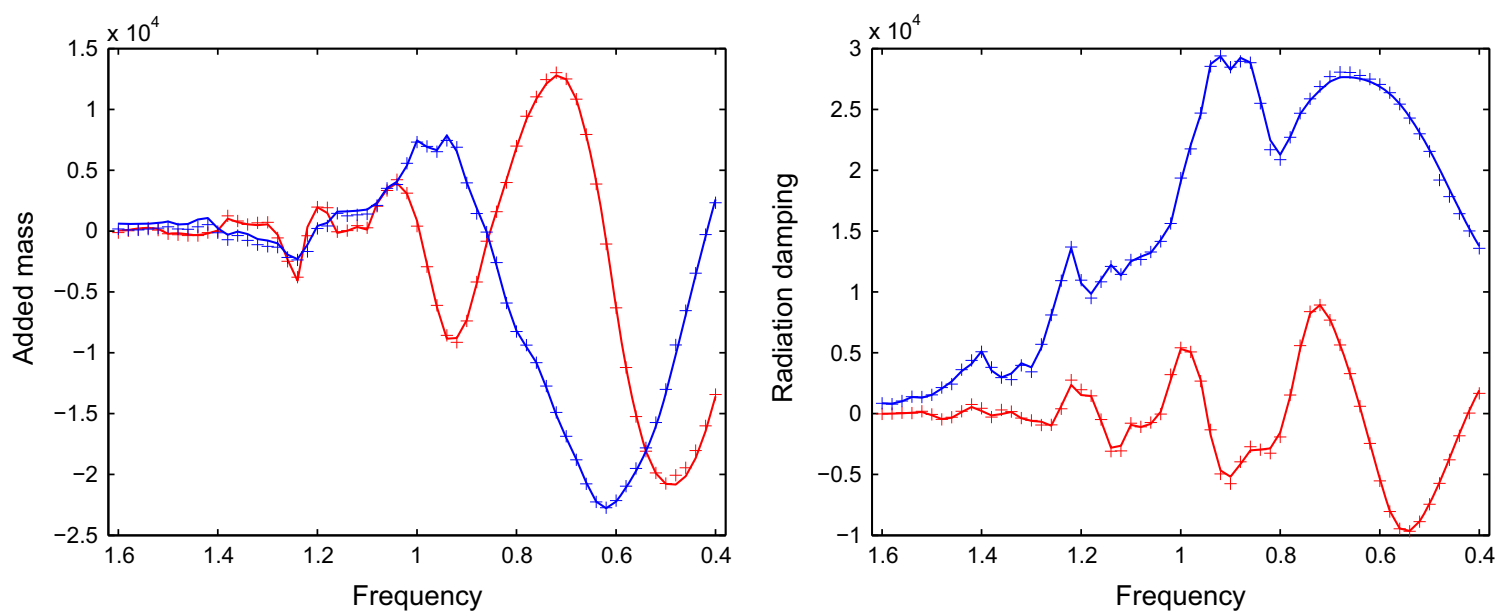

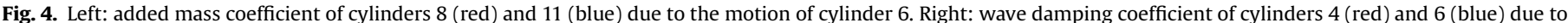
the motion of cylinder 6 . (For interpretation of the references to color in this figure caption, the reader is referred to the web version of this paper.) 
surging rectangular flaps were chosen. Each cylinder in the array has diameter and draft $10 \mathrm{~m}$ and each rectangular flap has length $10 \mathrm{~m}$ and draft and width $7.5 \mathrm{~m}$. These are typical values for prototypes of oscillating WECs. Comparisons are made with CBEM for accuracy and computational time. All the numerical computations were performed on a Dell workstation with an Intel Xeon CPU E5520@2.27 GHz and 4 GB RAM.

\subsection{Heaving cylinders}

The cylinders are arranged in a staggered array with a separation of $50 \mathrm{~m}$ laterally, as shown in (2). The wetted surface of each cylinder is discretized with 240 panels and the direction of propagation of the incident waves is $\beta=0$. The amplitudes of the heave excitation force computed by the proposed approach and by CBEM are shown in Fig. 3. In all cases, the results obtained from CBEM are marked with '+', and the results of our proposed approach are indicated by a line. The computations are carried out in the frequency range [0.4-1.6] rad s $\mathrm{s}^{-1}$, keeping in mind that most of the energy absorption through a WEC occurs in this range. The added mass and damping coefficients are plotted in Fig. 4 for

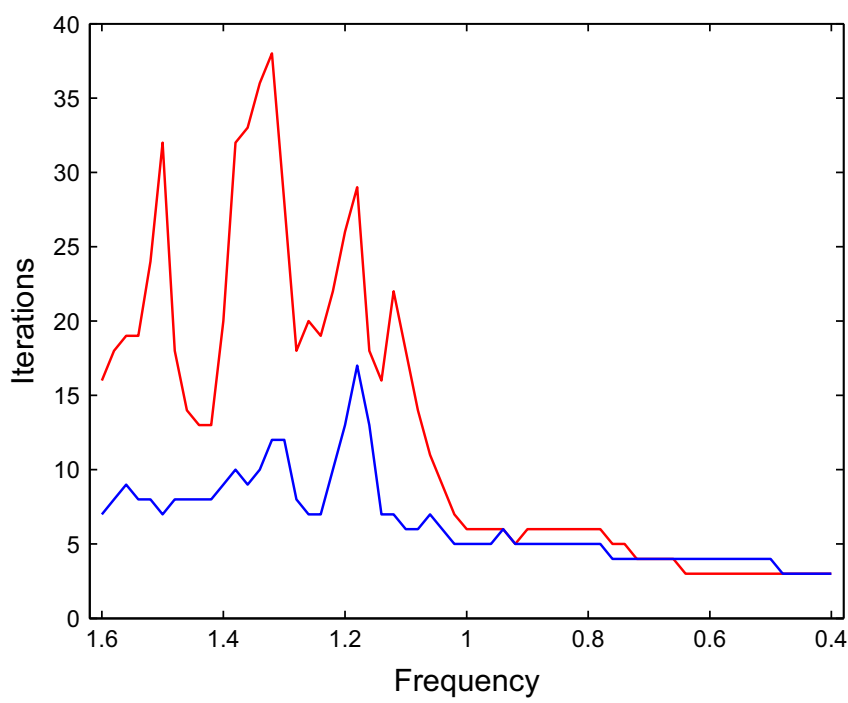

Fig. 5. Number of iterations required for convergence of the diffraction (red) and radiation (blue) problems. (For interpretation of the references to color in this figure caption, the reader is referred to the web version of this paper.) arbitrarily selected cylinders. In terms of computational time, the present approach took 20 min, whereas the CBEM took more than $140 \mathrm{~min}$ to solve for 61 frequencies. The number of iterations required for convergence in the proposed approach for the diffraction and radiation problems (when the cylinder at the center of the array is in motion) is shown in Fig. 5. As expected, the number of iterations required decreases with decreasing frequency.

\subsection{Surging rectangular flaps}

In this example, the array consists of 25 flaps arranged in a regular array with separating distance of $100 \mathrm{~m}$, shown in Fig. 6 . Each flap is discretized with 272 panels and the direction of propagation of the incident waves is $\beta=0$. The amplitude of the surge exciting force on selected flaps is shown in Fig. 7. The phase of excitation force on arbitrarily selected bodies is shown in Fig. 8. The added mass and wave damping coefficients are plotted in Fig. 9. For 61 frequencies, the CBEM required $190 \mathrm{~min}$, whereas the proposed approach took only $9 \mathrm{~min}$. The convergence pattern for diffraction and radiation problems is shown in Fig. 10. The number of iterations required for this array configuration was less than that of heaving cylinders, suggesting that the number of iterations decreases as the separating distance increases.

\subsection{Impact of separating distance on accuracy and computational time}

To investigate the impact of the separating distance on the overall accuracy of the computations, we consider two arrays consisting of 9 heaving cylinders and 9 surging flaps arranged in a regular array with distance ranging from 25 to $150 \mathrm{~m}$. The relative errors in the calculation of the excitation forces using the proposed approach

Error $=\frac{\left|F_{e x}^{C B E M}-F_{e x}^{p r e s e n t}\right|}{\left|F_{e x}^{C B E M}\right|}$,

computed at one frequency ( $1.2 \mathrm{rad} \mathrm{s}^{-1}$ ) for the body at the center of the array are plotted as a function of separating distance in Fig. 11. As expected, the error decreases as the separating distance increases, dropping to less than $5 \%$ when the separating distance is greater than five times the characteristic dimension of the body.

Further, to show that the approach is useful for larger arrays, we assess the computational time required by the present

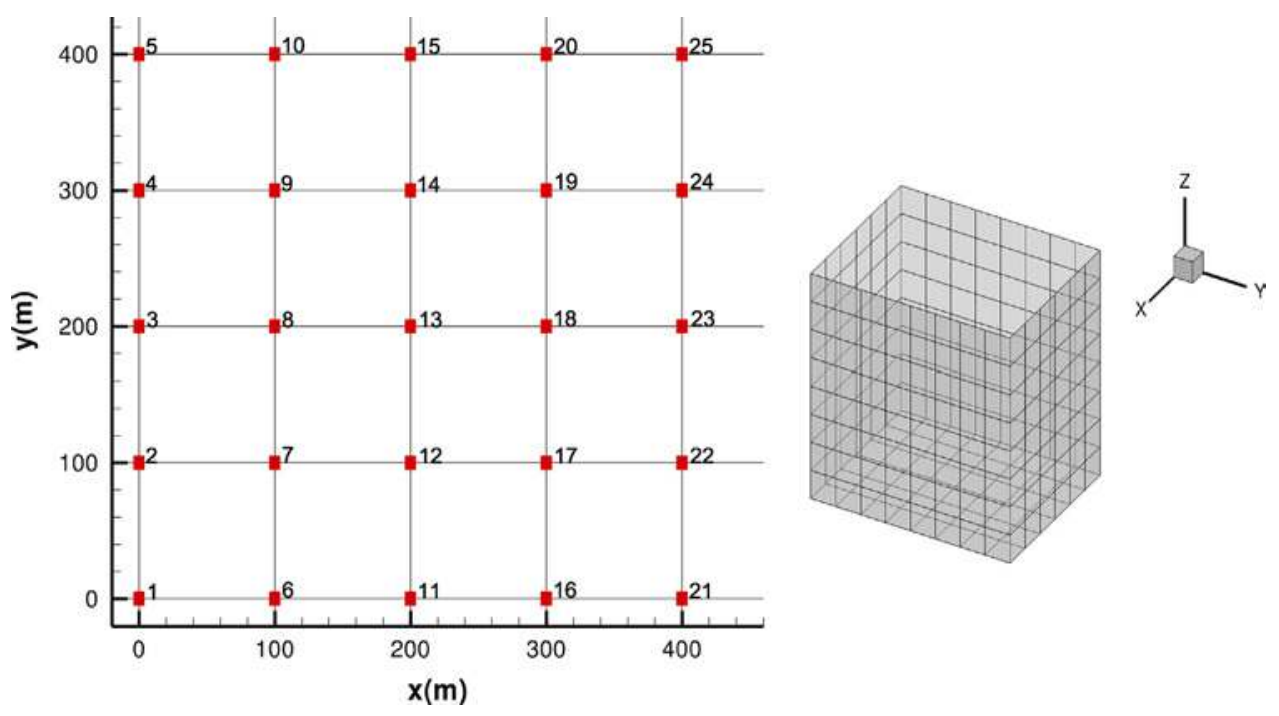

Fig. 6. Top view of array layout and surface mesh representation of a rectangular flap. 

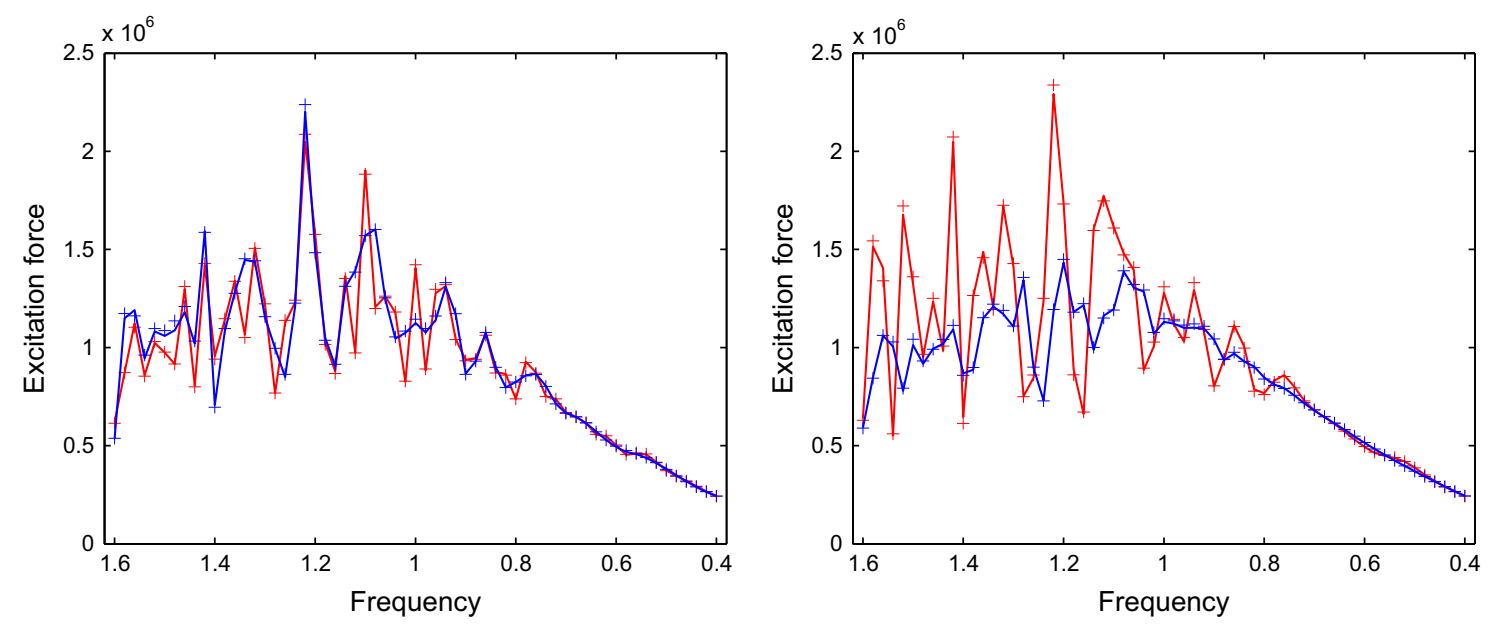

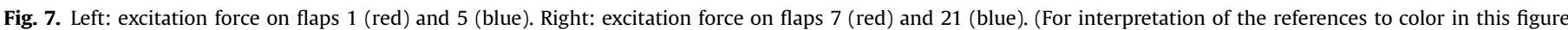
caption, the reader is referred to the web version of this paper.)
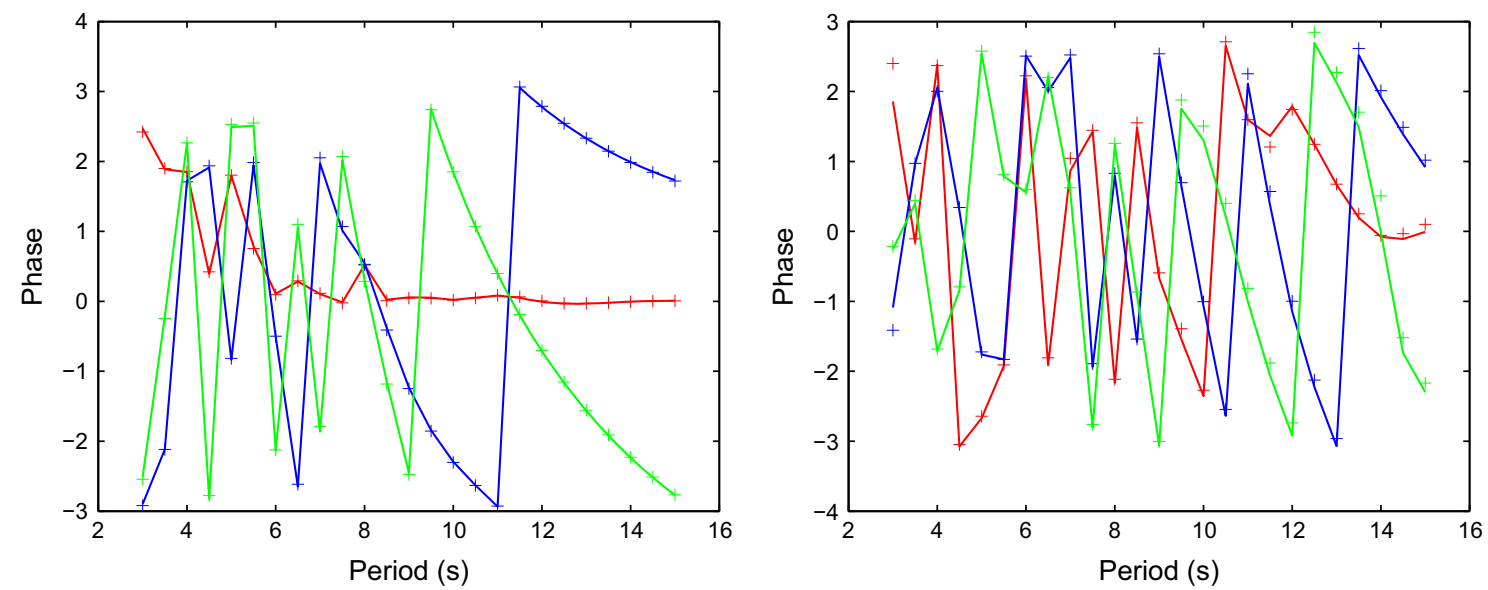

Fig. 8. Phase of excitation force on arbitrarily selected bodies in an array of 25 rectangular flaps.
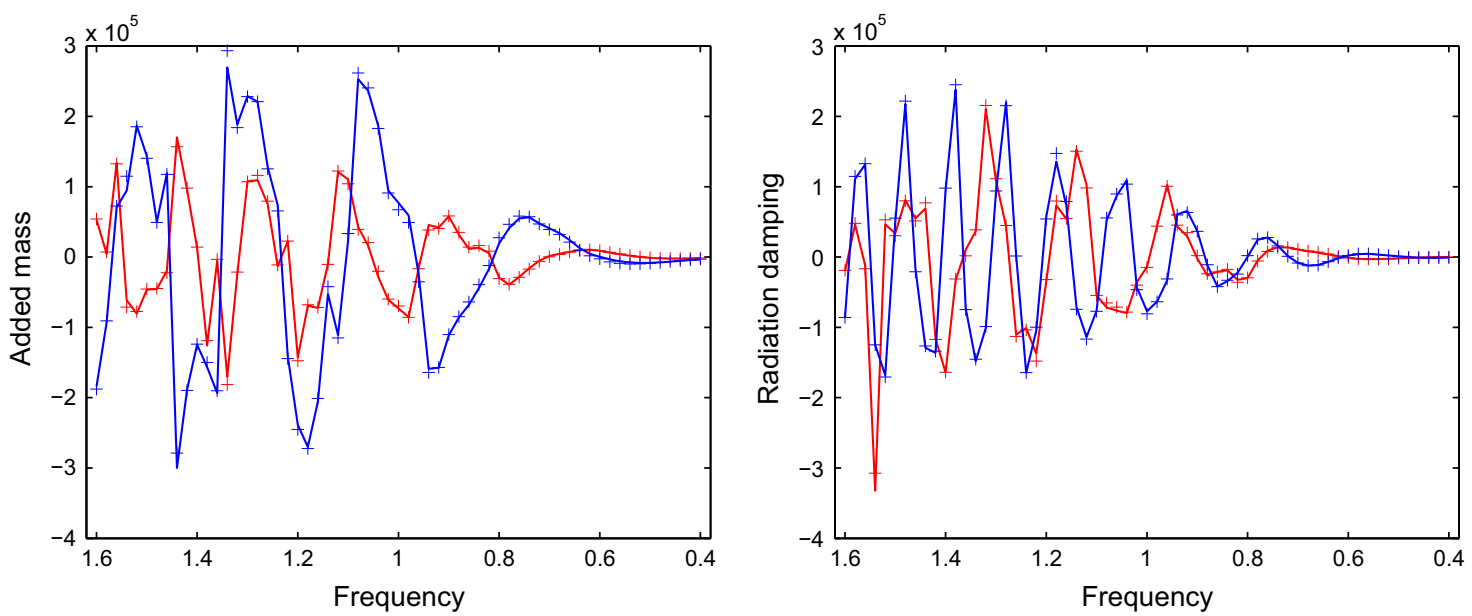

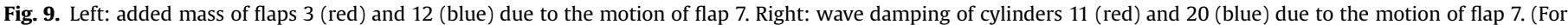
interpretation of the references to color in this figure caption, the reader is referred to the web version of this paper.)

approach as a function of the number of bodies. For simplicity, the layout of the cylinders is again in a regular array. The comparison of computational time using the present approach and CBEM is shown in Fig. 12. For CBEM, the computational time is simply the CPU time required to solve for one wave period using the direct Gauss solver, whereas for the present approach we plot the average time over 12 wave periods. This is because the number of iterations required to achieve convergence depends on the period, as shown in Figs. 5 and 10. Since we use a direct solver, the CBEM shows third order complexity, while the present approach is slightly less than first order complexity. This is because we store the inverse of the matrix computed in the first iteration and use it in subsequent iterations. We may point out that the memory requirements using the present approach are less than that of 


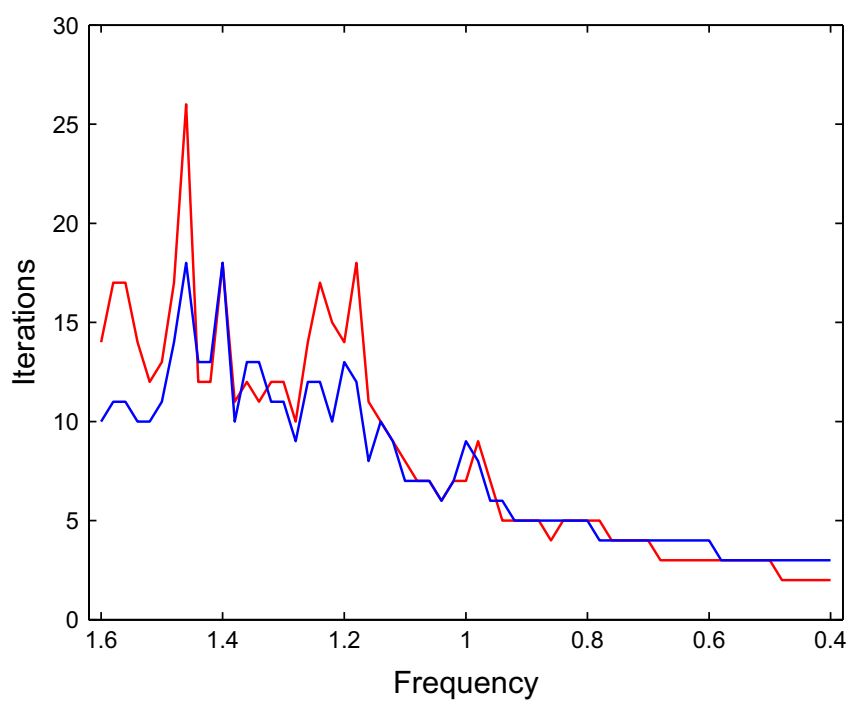

Fig. 10. Number of iterations required for convergence of the diffraction (red) and radiation (blue) problems. (For interpretation of the references to color in this figure caption, the reader is referred to the web version of this paper.)

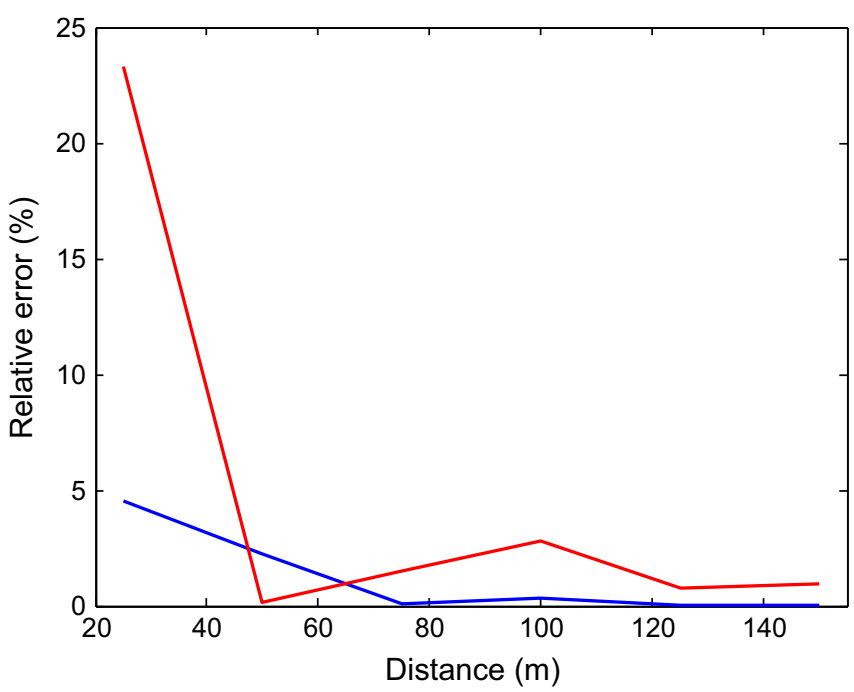

Fig. 11. Error in calculation of the surge (red) and heave (blue) excitation forces. (For interpretation of the references to color in this figure caption, the reader is referred to the web version of this paper.)

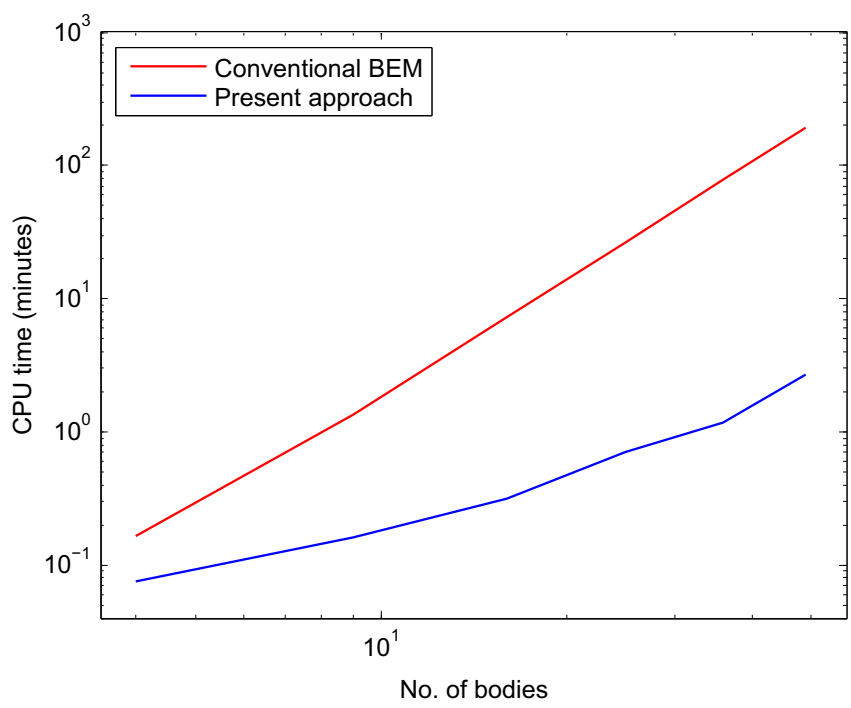

Fig. 12. CPU time comparison of conventional BEM and the present approach.
CBEM even when we store the influence coefficients corresponding to a single body.

\section{Wave interaction effects on power production}

The power production by an array of WECs is usually altered by the wave interaction effects (also called park effects), depending on the type of WEC, array layout, PTO mechanism, etc. Assessing the effects of wave interactions is thus critical when designing a WEC array; c.f. Babarit (2013) for a recent review.

We first consider power production by an array of 16 WECs, again to validate our approach against the results obtained by CBEM. The devices are surging rectangular flaps of the same dimensions as in the previous example, and they are arranged in a regular array (four rows with four WECs in each row) with a separating distance of $100 \mathrm{~m}$ in lateral directions. The stiffness and damping PTO characteristics of the flaps are $K_{\text {РТО }}=1402.1 \mathrm{kN} \mathrm{m}^{-1}$ and $B_{\text {РтО }}=444.2 \mathrm{kNs} \mathrm{m}^{-1}$, respectively. The damping PTO coefficient corresponds to the highest power production of an isolated WEC over the year. The wave data statistics measured at Yeu island on the French Atlantic coast are used (Fig. 13). Table 1 shows the annual average power for each row of flaps; row 1 contains those bodies that will face the incident waves first and so on. Good agreement is obtained between the results of proposed approach and CBEM.

We now consider arrays of 50 surging flap WECs. The purpose is to investigate the impact on power production of separating distance and number of rows, in both regular and staggered arrays. In doing so, our objective is to find an optimal array layout. Benefiting from previous studies we try to find such a layout while keeping the number of rows to a minimum; therefore, the number of rows is limited to either two (25 WECs in each row) or three $(17,16,17$ WECs in rows $1,2,3$ respectively). Further

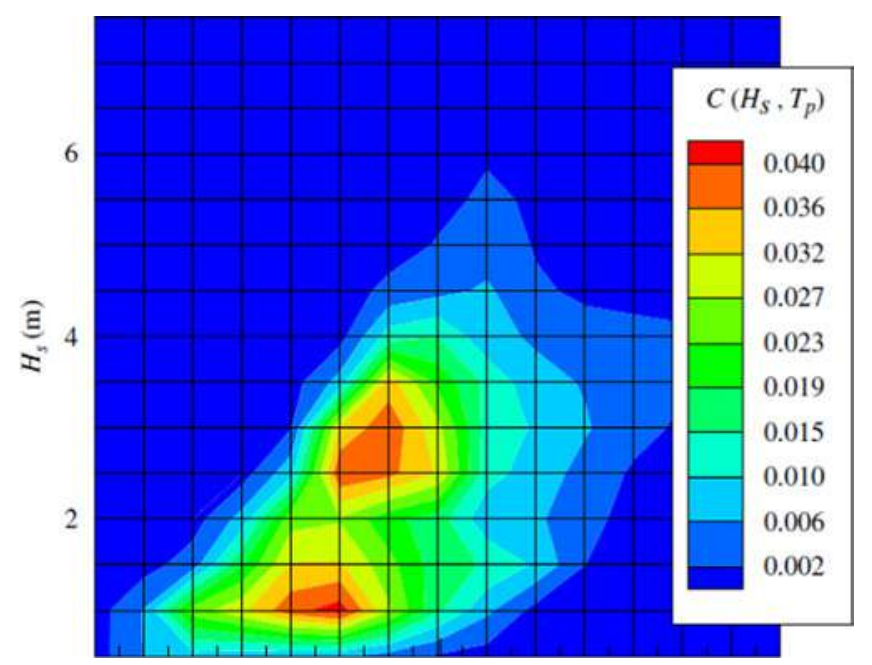

Fig. 13. Wave scatter diagram for Yeu island. Color layers: probability of occurrence $C\left(H_{s}, T_{p}\right)$. (For interpretation of the references to color in this figure caption, the reader is referred to the web version of this paper.)

Table 1

Annual average power $(\mathrm{kW})$ produced by a regular array of 16 WECs.

\begin{tabular}{lcc} 
& Present method & Conventional BEM \\
\hline Row 1 & 136.50 & 137.05 \\
Row 2 & 120.93 & 119.29 \\
Row 3 & 99.95 & 99.08 \\
Row 4 & 84.31 & 83.68 \\
\hline
\end{tabular}



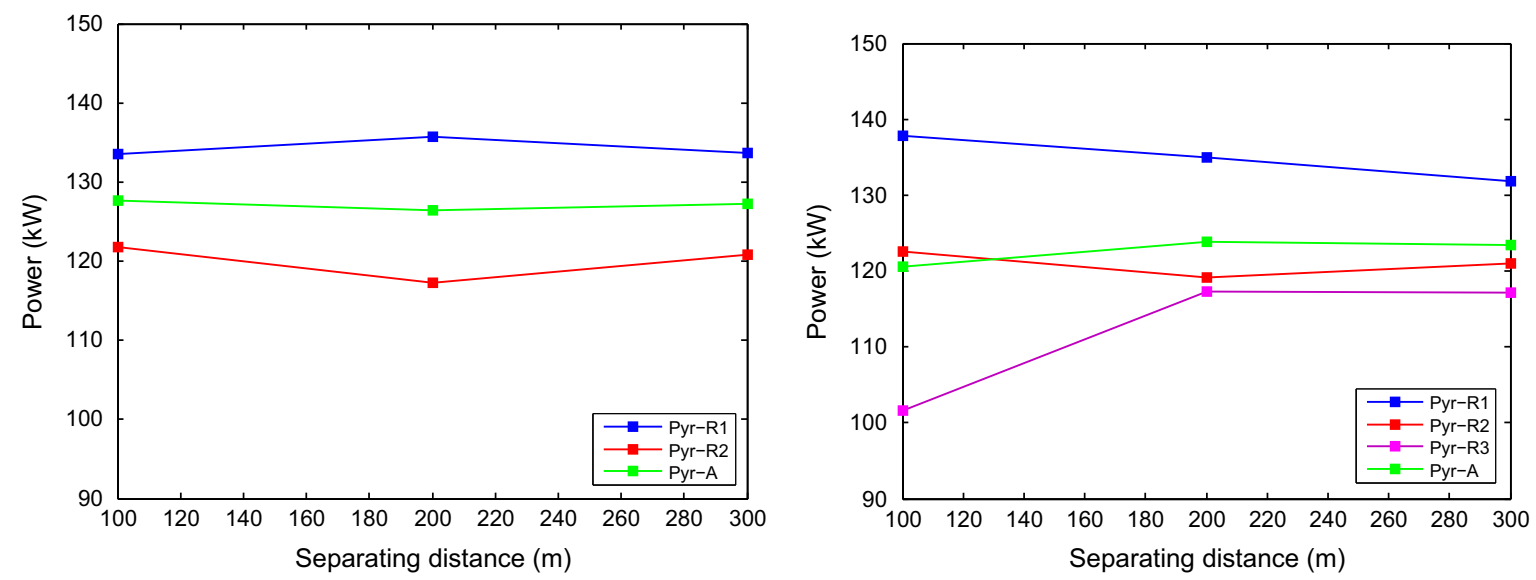

Fig. 14. Power production as a function of separating distance by regular arrays with two rows (left) and three rows (right).
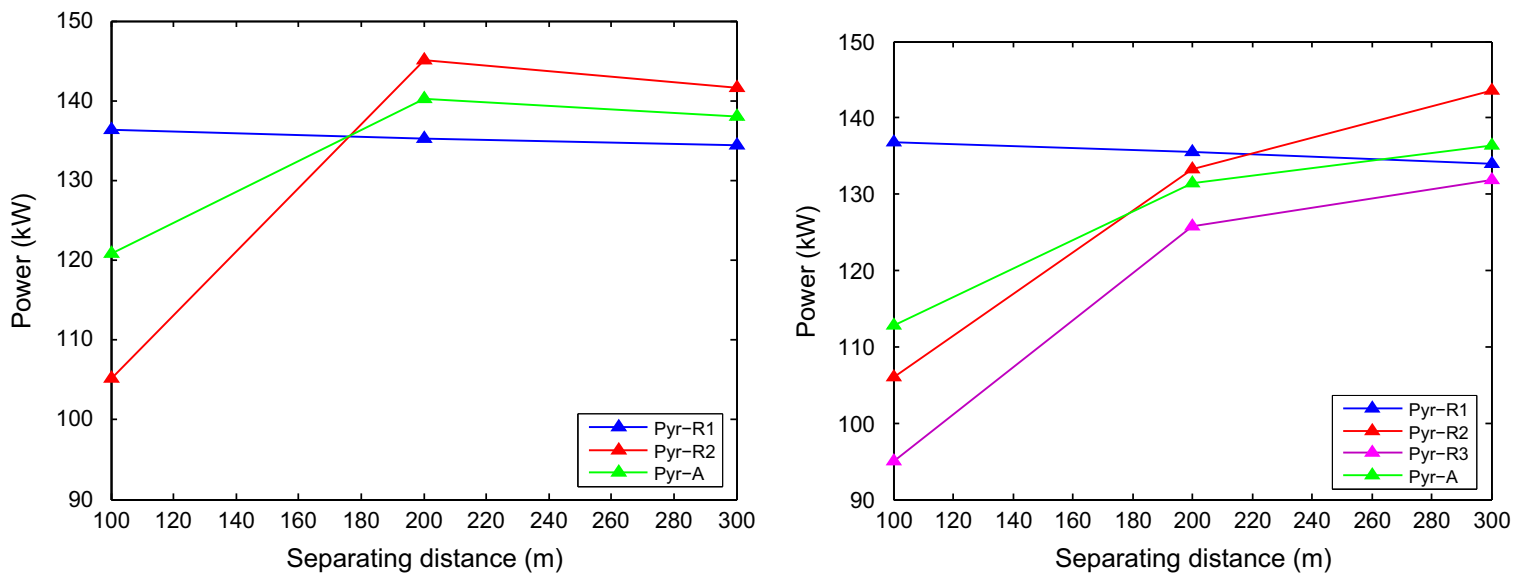

Fig. 15. Power production as a function of separating distance in staggered arrays with two (left) and three rows (right).

increasing the number of rows has a negative impact on power production, in both regular and staggered arrays, and hence is not considered here. In a single row array, the average power production by a single device is $132.92 \mathrm{~kW}$. There is negligible difference when the separating distance is increased. For reference, the power production by a single WEC is $130.6 \mathrm{~kW}$.

\subsection{Regular arrays}

The results for annual average power per device for two and three row arrays are shown in Fig. 14. The notations used are as follows: Pyr-R1 is the annual average power produced by a device in Row 1, and Pyr-A is the annual average power produced by all WECs in the array. The first row in both two and three row arrays benefits from the wave interactions from the rows at the back. This effect occurs for all values of separating distance, but decreases as separating distance increases. Addition of a row decreases the overall power production, particularly at short distances, with the effect mitigated by increasing distance. The results corroborate previous studies, which suggested that the power produced by the row at the back is less than that at the front (Borgarino et al., 2012). It is also worth noting that the wave interaction effects on power production decrease with the square root of the separating distance (as opposed to separating distance per se). Therefore, it can be noted that after a point, increasing the distance slightly increases the power production.

\subsection{Staggered arrays}

The results for yearly average power production for two and three row arrays are shown in Fig. 15. There are notable differences in the wave interaction effects in staggered arrays as compared to regular arrays. For small separating distances, the wave interaction effects are significantly destructive, with $8 \%$ less power production than in the regular array. The rows at the back (rows 2 and 3) suffer from destructive wave interaction effects. After increasing the separating distance to $200 \mathrm{~m}$, the interaction effects are clearly constructive in both arrays: rows at the back gain significantly, with an increment of $30-40 \%$. Similar to the regular array, we can observe that after a point, further increases in separating distance do not modify the power production significantly. Moreover, the power production is highest for a two-row array when the separating distance is $200 \mathrm{~m}$.

\section{Conclusions}

We have proposed a computational approach based on BEM and PWA to investigate wave interaction effects in sparse arrays of multiple bodies. The PWA allows us to split the full hydrodynamic problem into a sequence of smaller ones that are defined for an isolated body. The BEM is used to compute the contribution of an isolated body, while interaction effects are taken into account in an iterative manner. In each iteration, the wave interaction effects on a body from all other bodies in the array are approximated by a 
plane wave with appropriately chosen amplitude. It has been shown that the accuracy of such an approximation depends upon the separating distance between the devices: it is valid when the bodies are sufficiently far apart from each other. Our numerical experiments suggest that when the separating distance is greater than five times the characteristic dimension of the body, the approach provides reasonably accurate results for a wide range of frequencies. This is consistent with the results obtained when PWA alone is used to solve the hydrodynamic problems. In addition, due to the decomposition strategy, the computational resource requirements are significantly reduced. We were able to study the impact of wave interaction effects on the power production in arrays consisting of up to 50 WECs, which is difficult, if not impossible, using conventional BEM. Our findings related to the interaction effects on power production by arrays consisting of varying number of devices and different array layouts can be summarized as follows:

- It is economical to limit the number of rows to two or three.

- Up to a point, increasing the separating distance increases the power production; however, after this point, further increases in power production are small. For a 50 WEC array in a staggered layout, this distance is $200 \mathrm{~m}$. This means that further increasing the separating distance will only contribute to additional cable cost.

- The staggered arrays appear to be more profitable than regular arrays for the surging flap type of WEC.

- It is advisable to find typical array layouts for which wave interaction effects can be constructive.

\section{References}

Babarit, A., 2010. Impact of long separating distances on the energy production of two interacting wave energy converters. Ocean Eng. 37 (8), 718-729.

Babarit, A., 2013. On the park effect in arrays of oscillating wave energy converters. Renew. Energy 58, 68-78.

Borgarino, B., Babarit, A., Ferrant, P., 2011. An implementation of the fast multipole algorithm for wave interaction problems on sparse arrays of floating bodies. J. Eng. Math. 23, 1-18.
Borgarino, B., Babarit, A., Ferrant, P., 2012. Impact of wave interactions effects on energy absorption in large arrays of wave energy converters. Ocean Eng. 41, $79-88$.

Budal, K., 1977. Theory for absorption of wave power by a system of interacting bodies. J. Ship Res. 21 (4), 248-253.

Cruz, J., Sykes, R., Siddorn, P., Taylor, R.E., 2009. Wave farm design: preliminary studies on the influences of wave climate, array layout and farm control. In: Proceedings of the 8th European Wave and Tidal Energy Conference. pp. 736745.

Delhommeau, G., 1993. Seakeeping codes aquadyn and aquaplus. In: 19th WEGMENT School, Numerical Simulation of Hydrodynamics: Ship and Offshore Structures.

Falcãode, A.F.O., 2002. Wave-power absorption by a periodic linear array of oscillating water columns. Ocean Eng. 29 (10), 1163-1186.

Greengard, L., Rokhlin, V., 1987. A fast algorithm for particle simulations. J. Comput. Phys. 73 (2), 325-348.

Kagemoto, H., Yue, D., 1986. Interactions among multiple three-dimensional bodies in water waves: an exact algebraic method. J. Fluid Mech. 166 (1), 189-209.

Kashiwagi, M., 2000. Hydrodynamic interactions among a great number of columns supporting a very large flexible structure. J. Fluids Struct. 14 (7), 1013-1034.

Kring, D., Korsmeyer, T., Singer, J., White, J., 2000. Analyzing mobile offshore bases using accelerated boundary-element methods. Mar. Struct. 13 (4), 301-313.

Mavrakos, S., McIver, P., 1997. Comparison of methods for computing hydrodynamic characteristics of arrays of wave power devices. Appl. Ocean Res. 19 (5), 283-291.

Mclver, P., Evans, D., 1984. Approximation of wave forces on cylinder arrays. Appl Ocean Res. 6 (2), 101-107.

Newman, J., 1985. Algorithms for the free-surface Green function. J. Eng. Math. 19 (1) 57-67.

Ohkusu, M., 1974. Hydrodynamic forces on multiple cylinders in waves. In: International Symposium on the Dynamics of Marine Vehicles and Structures in Waves. pp. 107-112.

Peter, M.A., Meylan, M.H., 2004. Infinite-depth interaction theory for arbitrary floating bodies applied to wave forcing of ice floes. J. Fluid Mech. 500, 145-167.

Phillips, J., White, J., 1997. A precorrected-FFT method for electrostatic analysis of complicated 3-d structures. IEEE Trans. Comput. Aided Des. Integr. Circuits Syst. 16 (10), 1059-1072.

Ricci, P., Saulnier, J.-B., Falcão, A.F.O., 2007. Point-absorber arrays: a configuration study off the portuguese west-coast. In: Proceedings of 7th European Wave and Tidal Energy Conference.

Simon, M., 1982. Multiple scattering in arrays of axisymmetric wave-energy devices. part 1: a matrix method using a plane-wave approximation. J. Fluid Mech. 120, 1-25.

Teng, B., Gou, Y., 2006. Fast multipole expansion method and its application in bem for wave diffraction and radiation. In: Proceedings of the 16th International Offshore and Polar Engineering Conference. pp. 318-325.

Utsunomiya, T., Watanabe, E., 2002. Accelerated higher order boundary element method for wave diffraction/radiation problems and its applications. In: Proceedings of the 12th International Offshore and Polar Engineering Conference. pp. 305-312. 